\title{
Voltage-Mode Third-Order Quadrature Sinusoidal Oscillator Using VDBAs
}

\author{
Kanhaiya Lal Pushkar \\ Department of Electronics and Communication Engineering, Maharaja Agrasen Institute of Technology, Rohini, India \\ Email: klpushkar17@gmail.com
}

How to cite this paper: Pushkar, K.L. (2017) Voltage-Mode Third-Order Quadrature Sinusoidal Oscillator Using VDBAs. Circuits and Systems, 8, 285-292. https://doi.org/10.4236/cs.2017.812021

Received: December 4, 2017

Accepted: December 25, 2017

Published: December 28, 2017

Copyright $\odot 2017$ by author and Scientific Research Publishing Inc. This work is licensed under the Creative Commons Attribution International License (CC BY 4.0).

http://creativecommons.org/licenses/by/4.0/

\begin{abstract}
This paper presents a third-order quadrature sinusoidal oscillator (TOQSO) using two voltage differencing buffered amplifiers (VDBAs), three capacitors and a resistor. The new topology provides two quadrature voltage outputs. The condition of oscillation (CO) and frequency of oscillation (FO) are electronically independently controllable by the separate transconductance of the VDBAs. The workability of the proposed TOQSO is confirmed by SPICE (Version 16.5) simulation using Taiwan semiconductor manufacturing company (TSMC) $0.18 \mu \mathrm{m}$ process parameters.
\end{abstract}

\section{Keywords}

Voltage Differencing Buffered Amplifier, Third-Order Quadrature Sinusoidal Oscillator, Voltage-Mode

\section{Introduction}

An oscillator is a very important basic building block, which is frequently used in electrical and electronics engineering applications. Among several types of sinusoidal oscillators, the quadrature oscillators are widely used because they can offer sinusoidal signals with $90^{\circ}$ phase difference, for example, in telecommunications for quadrature mixers and SSB generators [1], for measurement purposes in vector generators and selective voltmeters [2]. Because of these applications number of QSOs has been realized employing different active building blocks in the open literature [3]-[8]. Different variety of active building blocks (ABBs) have been introduced in [9], VDBA is one of them. Since its introduction in [9], VDBA has been used in many signal processing and signal generation applications. Two biquad filters have been realized in [10] using two VDBAs and two capacitors and a resistor. In [11] VDBA based three lossless and lossy inductance 
simulators have been proposed employing two or three passive components. Single VDBA based multifunction filter configuration was proposed in [12] using five or six passive components. Quadrature oscillator employing three VDBAs, three capacitors and two resistors was proposed in [13]. The objective of this communication is to present a new voltage-mode TOQSO structure employing two VDBAs, three capacitors and a resistor which is based on a non-inverting VM low pass biquadratic filter and inverting VM integrator in a closed loop. The workability of the proposed configuration is verified by SPICE simulation using $0.18 \mu \mathrm{m}$ TSMC technology transistor parameters.

\section{Proposed Methodology}

Figure 1 shows the basic methodology to obtain a TOQSO by cascading a second-order non-inverting low pass filter with an inverting integrator or by cascading an inverting second-order low pass filter with non-inverting integrator. The open loop voltage gain of Figure 1 can be expressed as:

$$
\frac{V_{o}}{V_{\text {in }}}=T_{1}(s) T_{2}(s)=\frac{-a_{3}}{s\left(a_{0} s^{2}+a_{1} s+a_{2}\right)}
$$

where $T_{1}(s)=\frac{1}{a_{0} s^{2}+a_{1} s+a_{2}}$ and $T_{2}(s)=-\frac{a_{3}}{s}$

or $T_{1}(s)=-\frac{1}{a_{0} s^{2}+a_{1} s+a_{2}}$ and $T_{2}(s)=\frac{a_{3}}{s}$

To produced sustained oscillations, $V_{o}=V_{i n}$ and hence the characteristic equation can be denoted as

$$
a_{0} s^{3}+a_{1} s^{2}+a_{2} s+a_{3}=0
$$

The CO and FO can be deduced from Equation (2) as follows:

$$
\begin{gathered}
\mathrm{CO}: a_{0} a_{3}=a_{1} a_{2} \\
\text { FO }: \omega_{0}=\sqrt{\frac{a_{3}}{a_{1}}}=\sqrt{\frac{a_{2}}{a_{0}}}
\end{gathered}
$$

\section{The Proposed Third-Order Quadrature Oscillator Configuration}

The symbolic notation and equivalent model of the VDBA are shown in Figure 2(a) and Figure 2(b) respectively [9]. Using standard notations, the voltage-

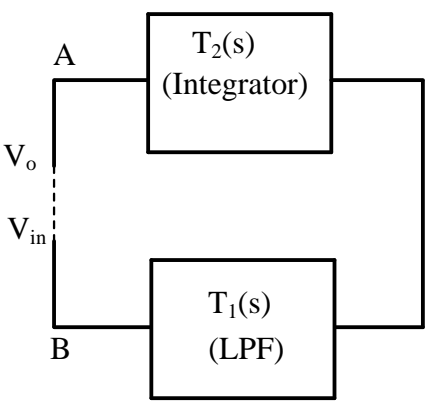

Figure 1. Functional block diagram for the realization of third-order quadrature oscillator. 


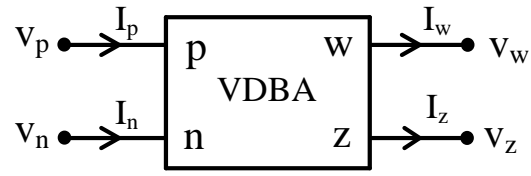

(a)

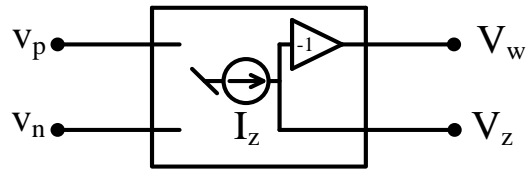

(b)

Figure 2. (a) Symbolic notation (b) equivalent model of VDBA.

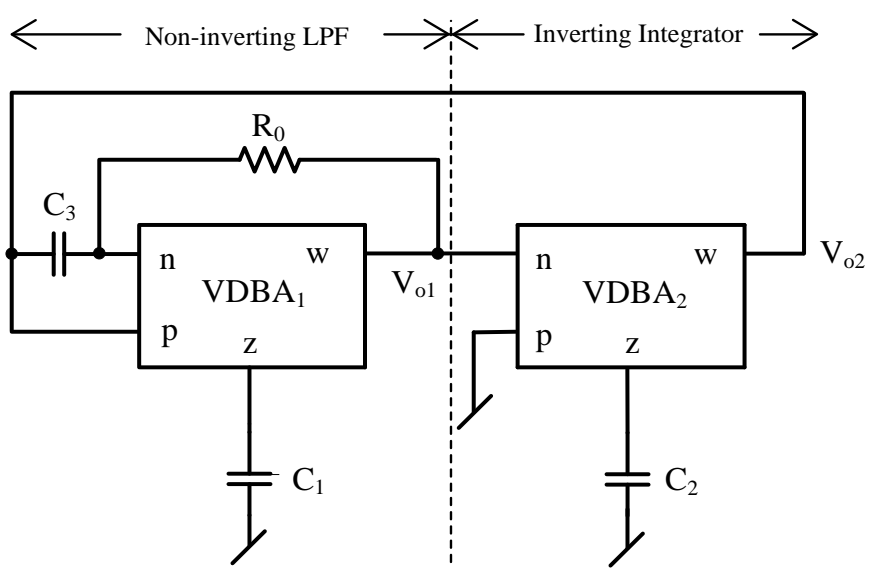

Figure 3. New proposed third-order quadrature sinusoidal oscillator.

current relations of VDBA can be described by the following matrix.

$$
\left(\begin{array}{c}
I_{p} \\
I_{n} \\
I_{z} \\
V_{w}
\end{array}\right)=\left(\begin{array}{cccc}
0 & 0 & 0 & 0 \\
0 & 0 & 0 & 0 \\
g_{m} & -g_{m} & 0 & 0 \\
0 & 0 & \beta & 0
\end{array}\right)\left(\begin{array}{c}
V_{p} \\
V_{n} \\
V_{z} \\
I_{w}
\end{array}\right)
$$

where $\beta$ is a non-ideal voltage gain of VDBA. The value of $\beta$ in an ideal VDBA is unity and $g_{m}$ is the transconductance of the VDBA.

Figure 3 shows the proposed new TOQSO with independent electronic control of both FO and CO.

The expression for characteristic equation (CE) of the circuit of Figure 3 is given by (Detailed explanation of Equation (6) with the help of Figure A1 is given in Appendix):

$$
\mathrm{CE}: s^{3} C_{1} C_{2} C_{3}+s^{2}\left(\frac{C_{1} C_{2}}{R_{0}}\right)+s\left(\frac{C_{2} g_{m 1}}{R_{0}}\right)+\frac{g_{m 1} g_{m 2}}{R_{0}}=0
$$

The condition of oscillation and the frequency of oscillation can be given as

$$
\begin{gathered}
\text { CO }: g_{m 2} R_{0} \leq \frac{C_{2}}{C_{3}} \\
\text { FO }: \omega_{0}=\sqrt{\frac{g_{m 1}}{R_{0} C_{1} C_{3}}}
\end{gathered}
$$

The relationship between $V_{o 1}$ and $V_{o 2}$ can be obtained as:

$$
\frac{V_{o 1}}{V_{o 2}}=\frac{-j \omega C_{2}}{g_{m_{2}}}=\frac{\omega C_{2}}{g_{m_{2}}} \mathrm{e}^{j-90^{\circ}}
$$


From Equation (9) it is evident that $V_{o 1}$ and $V_{o 2}$ are in quadrature.

\section{Sensitivity Analysis}

The sensitivity is an important performance criterion of any circuit structure. The sensitivities of $\omega_{0}$ with respect to active and passive elements are given by

$$
S_{C_{1}}^{\omega_{0}}=S_{C_{3}}^{\omega_{0}}=S_{R_{0}}^{\omega_{0}}=-\frac{1}{2}, S_{g_{m 1}}^{\omega_{0}}=\frac{1}{2}
$$

It may be easily observed from Equation (10) that all sensitivities are lower than unity in magnitude, for the proposed third-order quadrature oscillator. It ensures that the sensitivity performance is good.

\section{Simulation Results}

To confirm theoretical analysis, the proposed TOQSO was simulated using CMOS VDBA (as shown in Figure 4). The CMOS VDBA is implemented using $0.18 \mu \mathrm{m}$ TSMC real transistor models [14]. The aspect ratios of transistors used in Figure 4 are shown in Table 1. The passive elements were selected as $C_{1}=1.0$ $\mathrm{nF}$ and $C_{2}=1.0 \mathrm{nF}$, and $R_{0}=1.66 \mathrm{k} \Omega$. The transconductances of VDBAs were controlled by the bias currents. SPICE generated output waveforms indicating transient and steady state responses of circuit of Figure 3 are shown in Figure 5 and Figure 6 respectively. The results of TOQSO in Figure 5 and Figure 6 show more accuracy than the second order ones. These results, thus, shows the validity of the proposed configuration. Figure 7 shows the output spectrum of circuit

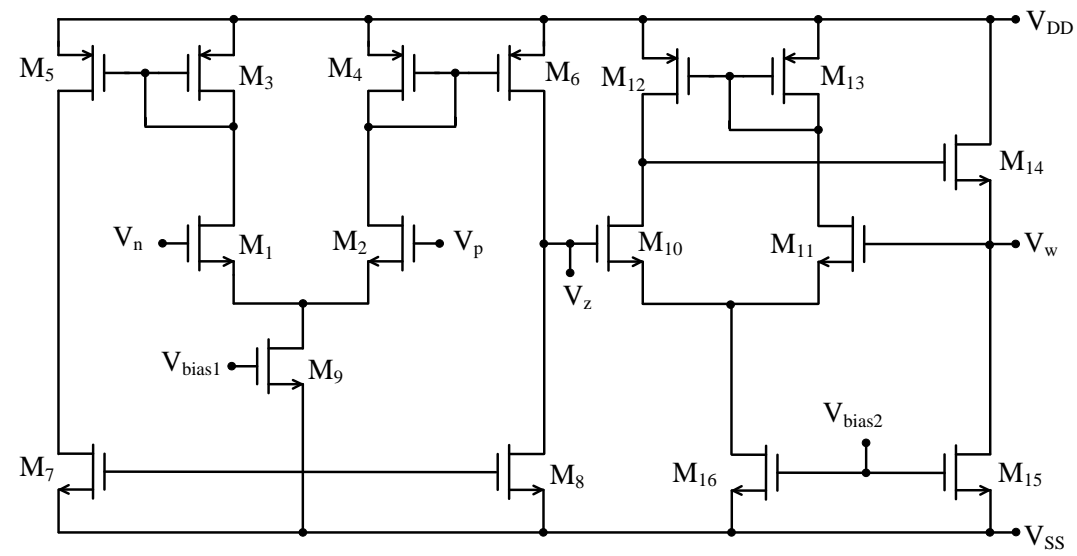

Figure 4. CMOS implementation of VDBA [10], $\mathrm{V}_{\mathrm{DD}}=\mathrm{V}_{\mathrm{SS}}=0.9 \mathrm{~V}$.

Table 1. The aspect ratios of transistors used in Figure 4.

\begin{tabular}{ccc}
\hline Transistor & $W(\mu \mathrm{m})$ & $L(\mu \mathrm{m})$ \\
\hline M1-M4, M10, M11, M15, M16 & 7 & 0.35 \\
M5, M6 & 21 & 0.7 \\
M7, M8 & 7 & 0.7 \\
M9 & 3.5 & 0.7 \\
M12-M14 & 14 & 0.35 \\
\hline
\end{tabular}




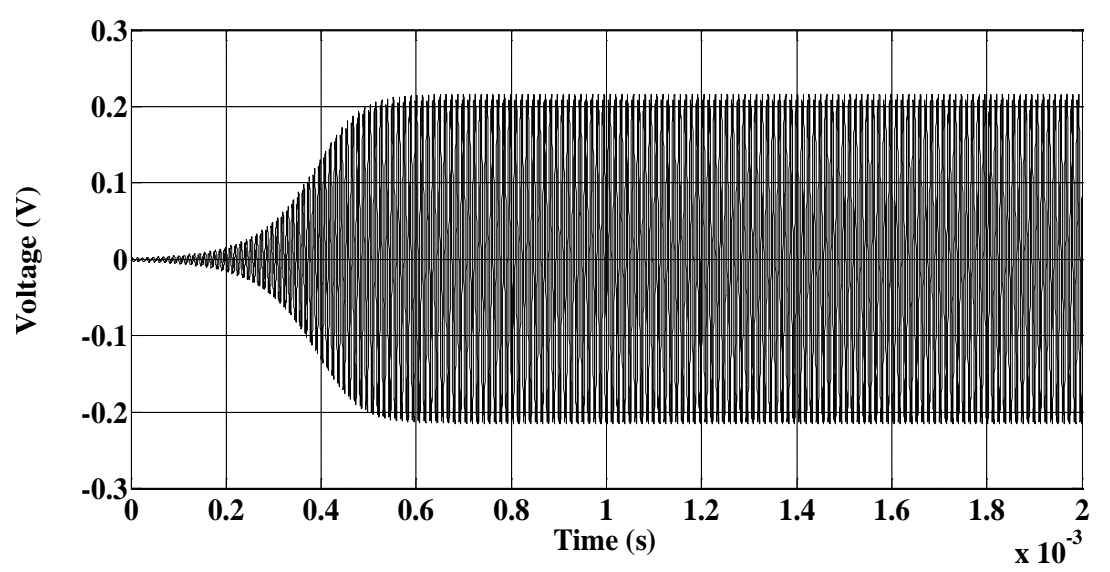

Figure 5. Transient response of proposed third-order quadrature sinusoidal oscillator.

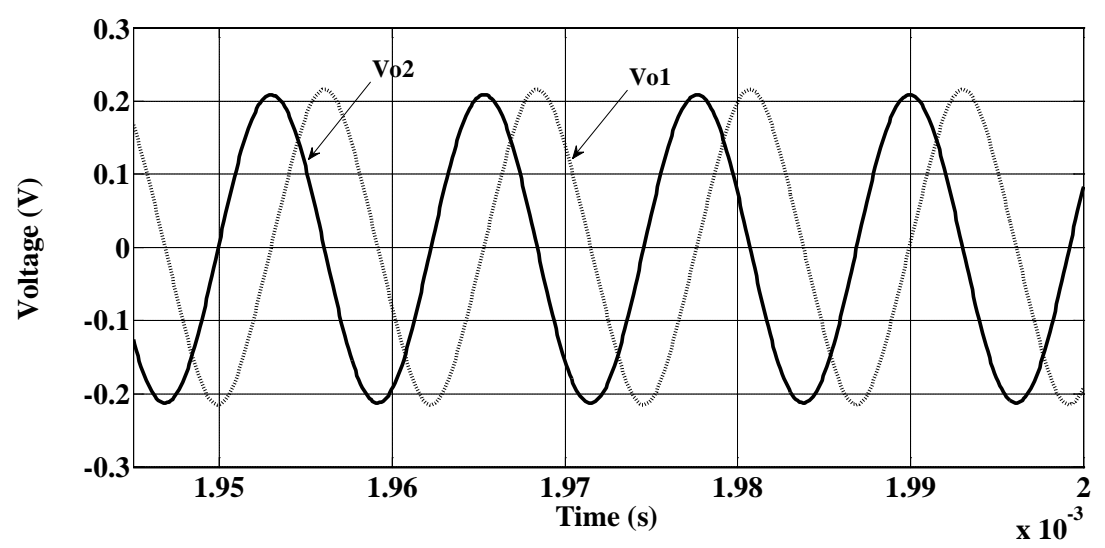

Figure 6. Study state response of proposed third-order quadrature sinusoidal oscillator.

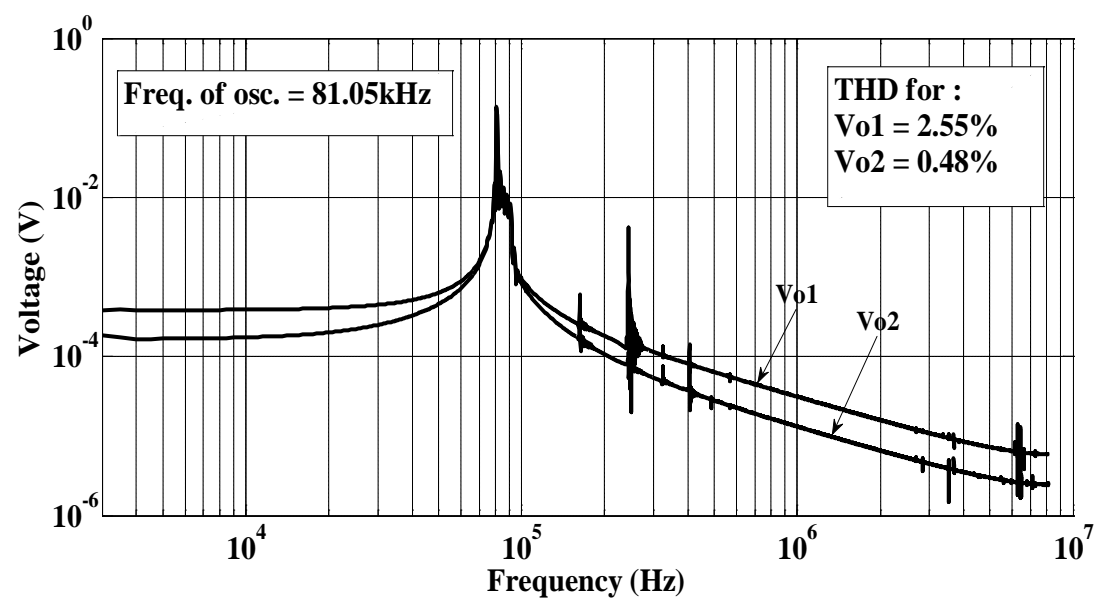

Figure 7. Frequancy response of proposed third-order quadrature sinusoidal oscillator.

shown in Figure 3; whereas the total harmonic distortion (THD) for both the outputs, $\mathrm{V}_{\mathrm{o} 1}$ and $\mathrm{V}_{\mathrm{o} 2}$ are found to be $2.55 \%$ and $0.48 \%$ respectively. The THD at output $\mathrm{V}_{\mathrm{o} 2}$ is very small. Figure 8 shows the Lissajous pattern for the circuit of Figure 3. The circles are shown in the Figure 8, indicates that two signals are at $90^{\circ}$ phase difference. 


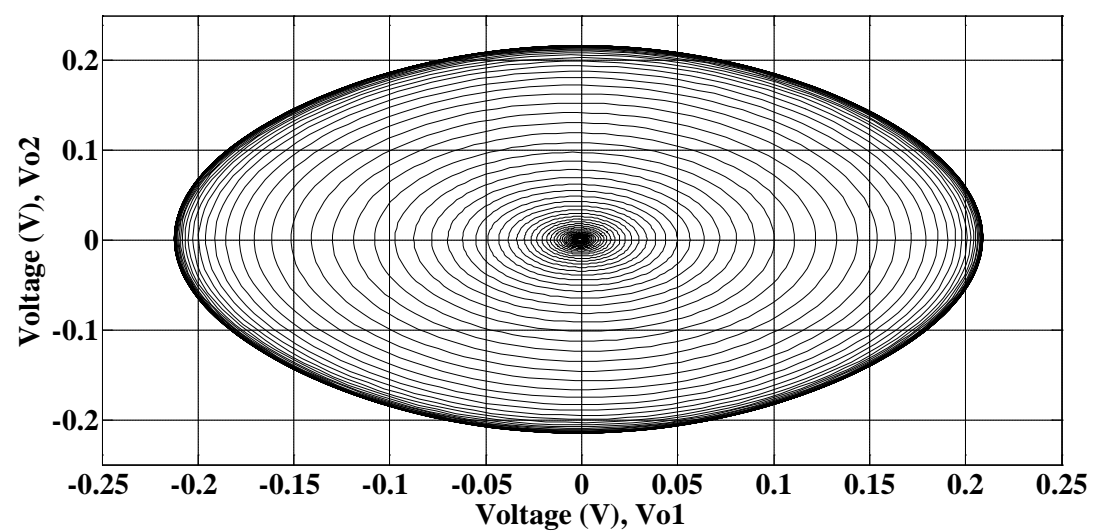

Figure 8. Frequancy response of proposed third-order quadrature sinusoidal oscillator.

\section{Conclusion}

A new voltage-mode third order quadrature sinusoidal oscillator with independent electronic control of both $\mathrm{CO}$ and FO using two VDBAs, three capacitors and a resistor is introduced. The $\mathrm{CO}$ can be electronically controlled by transcoductance $\left(g_{m 2}\right)$ of $\mathrm{VDBA}_{2}$ without affecting FO. FO can also be electronically adjusted by transcoductance $\left(g_{m 1}\right)$ of $\mathrm{VDBA}_{1}$ without affecting $\mathrm{CO}$. The proposed TOQSO offers low sensitivities. The circuit exhibits good high frequency performance. One can design TOQSO with single VDBA. Workability of the proposed configuration is verified by SPICE simulation using $0.18 \mu \mathrm{m}$ TSMC technology.

\section{References}

[1] Horng, J.W., Hou, C.L., Chang, C.M., Chung, W.Y., Tang, H.W. and Wen, Y.H. (2005) Quadrature Oscillators Using CCIIs. International Journal of Electronics, 92, 21-31. https://doi.org/10.1080/00207210412331332899

[2] Gibson, J.D. (1997) The Communication Handbook. CRC Press, Boca Raton, FL, USA, 1997.

[3] Tangsrirat, W. and Surakampontorn, W. (2090) Single-Resistance-Controlled Quadrature Oscillator and Universal Biquad Filter Using CFOAs. AEU - International Journal of Electronics and Communications, 63, 1080-1086. https://doi.org/10.1016/j.aeue.2008.08.006

[4] Horng, J.W. (2002) Current Differencing Buffered Amplifiers Based Single Resistance Controlled Quadrature Oscillator Employing Grounded Capacitors. IEICE Transactions on Fundamental of Electronics, Communications and Computer Sciences, E85-A, 1416-1419.

[5] Ozcan, S., Toker, A., Acar, C., Kuntman, H. and Cicekoglu, O. (2000) Single Resistance-Controlled Sinusoidal Oscillators Employing Current Differencing Buffered Amplifier. Microelectronics Journal, 31, 169-174. https://doi.org/10.1016/S0026-2692(99)00113-5

[6] Prommee, P. and Dejhan, K. (2002) An Integrable Electronic-Controlled Quadrature Sinusoidal Oscillator Using CMOS Operational Transconductance Amplifier. International Journal of Electronics, 89, 365-379. https://doi.org/10.1080/713810385

[7] Rodriguez-Vazquez, A., Linares-Barranco, B. Huertas, J. L. and Sanchez-Sinencio, 
E. (1990) On the Design of Voltage-Controlled Sinusoidal Oscillators Using OTAs. IEEE Transactions on Circuits and Systems, 37, 198-211.

https://doi.org/10.1109/31.45712

[8] Holzel, R. (1993) A Simple Wide-Band Sine Wave Quadrature Oscillator. IEEE Transactions on Instrumentation and Measurement, 42, 758-760. https://doi.org/10.1109/19.231604

[9] Biolek, D. Senani, R., Biolkova, V. and Kolka, Z., (2008) Active Elements for Analog Signal Processing: Classification, Review, and New Proposals. Radioengineering, 17, 15-32.

[10] Kacar, F. Yeil, A. and Noori, A. (2012) New CMOS Realization of Voltage Differencing Buffered Amplifier and Its Biquad Filter Applications. Radioengineering, 21, 333-379.

[11] Firat Kacar, A. Y. (2016) VDBA-Based Lossless and Lossy Inductance Simulators and Its Filter Applications. Signal Processing and Communication Application Conference (SIU) 24 ${ }^{\text {th }}$ 2016, Zonguldak, Turkey, 16-19 May 2016.

[12] Gupta, P. and Pandey R. (2017) Single VDBA Based Multifunction Filter. International Journal of Control Theory and Applications, 10, 651-661.

[13] Malhotra, C., Ahalawat, V. V., Kumar, V. Pandey, R. and Pandey, N. (2016) Voltage Differencing Buffered Amplifier Based Quadrature Oscillator. $1^{\text {st }}$ International Conference on Power Electronics, Intelligent Control and Energy Systems (ICPEICES-2016), Delhi, India, 4-6 July 2016. https://doi.org/10.1109/ICPEICES.2016.7853457

[14] Minaei, S and Yuce, E. (2010) Novel Voltage-Mode All-Pass Filter Based on Using DVCCs. Circuits System and Signal Processing, 29, 391-402.

https://doi.org/10.1007/s00034-010-9150-3 


\section{Appendix}

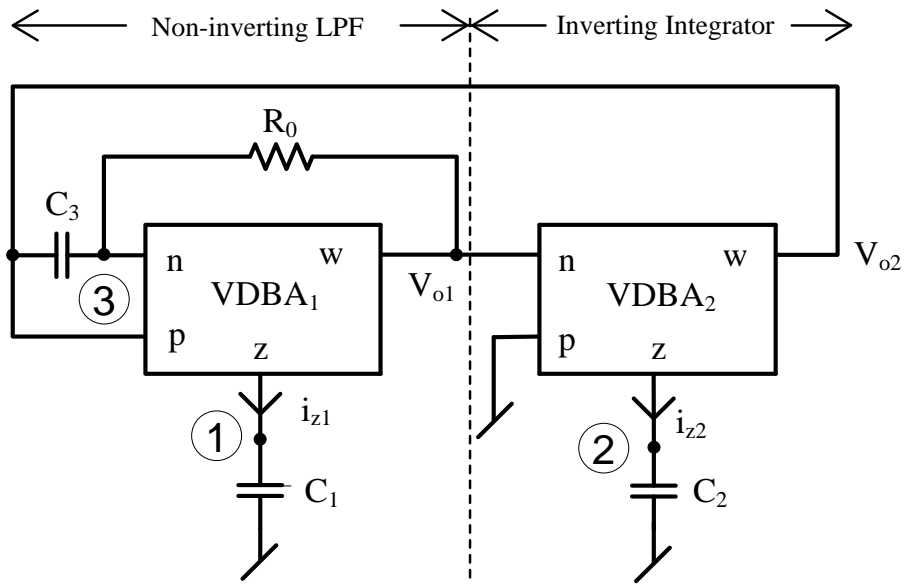

Figure A1. New proposed third-order quadrature sinusoidal oscillator.

KCL at node 1:

$$
\begin{gathered}
i_{z 1}=g_{m_{1}}\left(v_{p_{1}}-v_{n_{1}}\right)=v_{z_{1}} s C_{1} \\
g_{m_{1}}\left(v_{p_{1}}-v_{n_{1}}\right)=v_{z_{1}} s C_{1}
\end{gathered}
$$

KCL at node 2:

$$
\begin{aligned}
i_{z 2}= & g_{m_{2}}\left(0-v_{n_{2}}\right)=v_{z_{2}} s C_{2} \\
& -g_{m_{2}} v_{z_{1}}=v_{z_{2}} s C_{2},
\end{aligned}
$$

KCL at node 3:

$$
v_{n_{1}}\left(s C_{3}+\frac{1}{R_{0}}\right)=v_{z_{2}} s C_{3}+v_{z_{1}} \frac{1}{R_{0}}
$$

From Equations ((a)-(c)), we will gate CE. 